\title{
The effect of muscle fatigue on stimulus intensity requirements for central and peripheral fatigue quantification
}

\author{
Daria Neyroud • Alexia Vallotton • Guillaume Y. Millet • \\ Bengt Kayser • Nicolas Place
}

Received: 2 May 2013 / Accepted: 21 October 2013 / Published online: 7 November 2013

(C) Springer-Verlag Berlin Heidelberg 2013

\begin{abstract}
Purpose The present study was designed to determine the stimulation intensity necessary for an adequate assessment of central and peripheral components of neuromuscular fatigue of the knee extensors.

Methods Three different stimulation intensities (100, 120 and $150 \%$ of the lowest intensity evoking a plateau in M-waves and twitch amplitudes, optimal stimulation intensity, OSI) were used to assess voluntary activation level (VAL) as well as M-wave, twitch and doublet amplitudes before, during and after an incremental isometric exercise performed by 14 ( 8 men) healthy and physically active volunteers. A visual analog scale was used to evaluate the associated discomfort.

Results There was no difference $(p>0.05)$ in VAL between the three intensities before and after exercise. However, we found that stimulating at $100 \%$ OSI may overestimate the extent of peripheral fatigue during exercise, whereas $150 \%$ OSI stimulations led to greater discomfort associated with doublet stimulations as well as to an increased antagonist co-activation compared to $100 \%$ OSI.
\end{abstract}

Communicated by Toshio Moritani.

D. Neyroud

Institute of Movement Sciences and Sports Medicine,

University of Geneva, Geneva, Switzerland

D. Neyroud · A. Vallotton · B. Kayser · N. Place $(\triangle)$ Institute of Sport Sciences and Department of Physiology,

Faculty of Biology and Medicine, University of Lausanne,

Quartier UNIL-Mouline, Building Geopolis,

Lausanne, Switzerland

e-mail: Nicolas.Place@unil.ch

G. Y. Millet

Université de Lyon, 42023 Saint-Étienne, France
Conclusion We recommend using $120 \%$ OSI, as it constitutes a good trade-off between discomfort and reliable measurements.

Keywords Interpolated twitch technique $\cdot$ Peripheral fatigue - Isometric contraction - Transcutaneous electrical stimulation

$\begin{array}{ll}\text { Abbreviations } \\ \text { BF } & \text { Biceps femoris } \\ \text { EMG } & \text { Electromyography } \\ \text { MVC } & \text { Maximal Voluntary Contraction } \\ \text { OSI } & \text { Optimal Stimulation Intensity } \\ \text { RF } & \text { Rectus femoris } \\ \text { RMS } & \text { Root Mean Square } \\ \text { RMS/M } & \text { RMS normalized by the M-wave amplitude } \\ \text { VAL } & \text { Voluntary Activation Level } \\ \text { VL } & \text { Vastus lateralis } \\ \text { VM } & \text { Vastus medialis }\end{array}$

\section{Introduction}

Central (neural) and peripheral (muscular) fatigues are routinely quantified by measuring the forces evoked by transcutaneous electrical stimulation of the main motor nerve trunk, applied while the muscle is relaxed or superimposed to a maximal voluntary contraction (MVC) - the twitch interpolation technique (Merton 1954). The extent of additional force elicited by stimulation during the MVC is used to describe the central component, even though it has been reported that it may overestimate voluntary drive and appear to be reliable only for high contraction intensities (Behm et al. 1996; Kooistra et al. 2007), while the loss of twitch force of relaxed muscle is used to describe 
the peripheral component of neuromuscular fatigue (Merton 1954; Edwards et al. 1977; Bigland-Ritchie et al. 1986; Gandevia 2001; Millet and Lepers 2004; Place et al. 2010b; Millet et al. 2011a).

Nonetheless, no consensus exists on how to determine the stimulation intensity necessary to do this. It is generally accepted that the optimal stimulation intensity (OSI) corresponds to the intensity needed for complete spatial recruitment of the muscle group of interest (Adam and De Luca 2005; Sogaard et al. 2006; Kooistra et al. 2007). For some authors, this intensity is reached when the evoked force does not rise anymore, despite an increase in stimulation intensity (Kubo et al. 2004; Adam and De Luca 2005; Sogaard et al. 2006), whereas for others it corresponds to a plateau in the compound muscle action potential (M-wave) amplitude of the muscles considered (Hamada et al. 2000; Lévénez et al. 2005; Sidhu et al. 2009; Klass et al. 2012). Some studies used both force and M-wave amplitudes to determine OSI (Löscher et al. 1996; Hamada et al. 2000; Babault et al. 2001; Place et al. 2005; Zory et al. 2005; Gondin et al. 2006; Perrey et al. 2010). However, it is not known whether such stimulation intensities are sufficient to allow complete spatial recruitment in the fatigued state. Whereas some researchers just used the OSI (Babault et al. 2001; Place et al. 2005; Wüst et al. 2008), others used higher intensities ranging from $110 \%$ (Babault et al. 2001; Adam and De Luca 2005; Zory et al. 2005) to $150 \%$ (Löscher et al. 1996; Lagerquist and Collins 2010) OSI.

There are several reasons for increasing stimulus intensity beyond OSI. Firstly, with sustained or repeated firing, there is an increase in the excitation threshold of the nerve fibers (Kernell and Monster 1982a, b; Vagg et al. 1998; Burke 2002; Butler et al. 2003). Secondly, during voluntary contractions, the stimulating electrode may shift due to anatomical configuration changes (Place et al. 2010a). For these reasons, OSI, as determined in the pre-exercise non-fatigued condition, may not be sufficient for complete spatial motor unit recruitment post exercise. On the other hand, there are also reasons to limit stimulus intensity. One reason for limiting the stimulation intensity is that discomfort associated with electrical stimulation is directly related to its intensity (Alon 1985; Gandevia 2001). High level of discomfort may prevent subjects to maximally contract their muscles (Button and Behm 2008). A further reason to limit stimulation intensity is that beyond a given intensity it may lead to the recruitment of antagonist muscles (Place et al. 2010a; Millet et al. 2012).

It thus appears that there is a trade-off to be made between the minimum stimulation intensity allowing a reliable evaluation of the state of muscle fatigue and a maximum tolerable intensity that does not activate antagonists or makes the subject anxious about the stimulation. We, therefore, evaluated different stimulation intensities at the femoral nerve level to assess central and peripheral fatigue of the knee extensors. We hypothesized that the OSI (determined in the fresh state by considering a plateau in both force and $\mathrm{M}$-wave amplitude despite a slight increase in stimulation intensity) would not be sufficient to maximally recruit the motoneurons in the fatigued state. Choosing an intensity beyond OSI would allow maximal recruitment and better assessment of fatigue, while a too elevated stimulation intensity would produce co-activation of antagonist muscles and higher discomfort, which could lead to depreciated force levels.

\section{Methods}

\section{Subjects}

Fourteen ( 8 men and 6 women) healthy and physically active volunteers $(26.4 \pm 3.7$ years, $173.1 \pm 7.4 \mathrm{~cm}$, $69.5 \pm 9.9 \mathrm{~kg}$ ) participated in the study after having been informed of the experimental procedures. The study protocol was approved by the Ethics Committee of the University Hospitals of Geneva (protocol 11-230) and was in agreement with the declaration of Helsinki. Each subject gave a written consent before participation.

\section{Experimental protocol}

The subjects were seen twice. On the first occasion, they were informed in detail about the procedures and risks. After giving their consent, they underwent a thorough familiarization session to get used to the procedures. During the familiarization session, subjects were trained to perform MVCs (about 5-10 attempts to obtain reliable MVCs, i.e., with no more than $5 \%$ variation between the last trials) as well as to sustain different force levels with visual feedback. They were also familiarized with single and double stimulations, the latter both at rest and superimposed to MVCs. On a separate occasion, all measurements were then obtained in one experimental 2-h long session (see Fig. 1). On subjects' arrival, after instrumentation with stimulation and recording electrodes, the optimal intensity for the electrical stimulation was determined by progressively increasing the stimulus intensity by $10 \mathrm{~mA}$ increments until no further increase in twitch force or M-waves amplitudes occurred (see below).

\section{Pre-fatigue neuromuscular assessment}

A single (twitch) and paired (doublet at $100 \mathrm{~Hz}$ ) stimulus were delivered at 100,120 and $150 \%$ OSI. The order in which the different stimulation intensities were evoked 

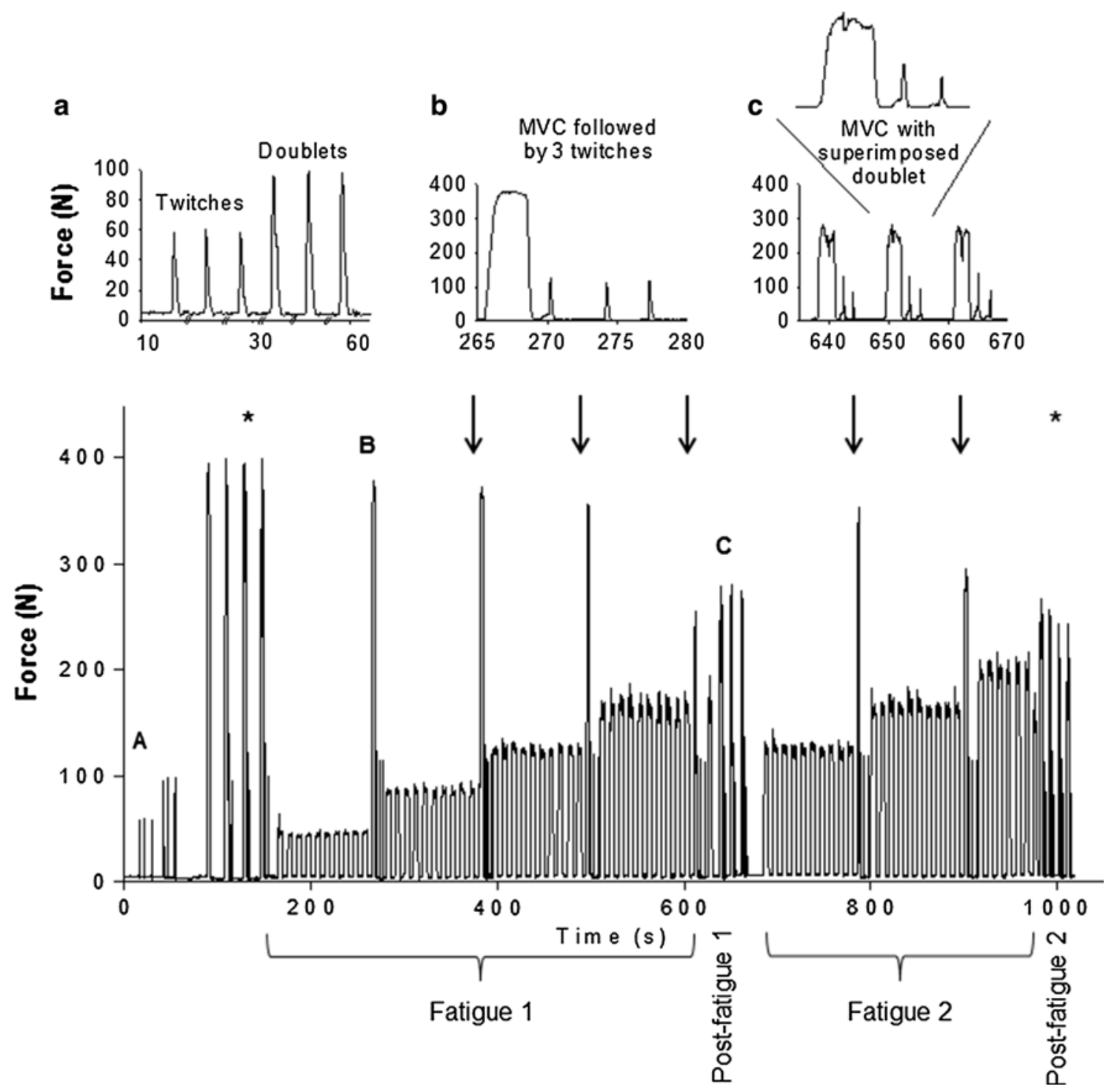

Fig. 1 Protocol design. a Typical twitches and doublets at the three different intensities (please note that the time axis has been broken for clarity), $\mathbf{b}$ a typical MVC performed at the end of each set and the three following doublets evoked at the three different intensities, and c typical maximal voluntary contractions (MVCs) with superimposed

was counterbalanced between subjects, but kept constant for a given subject. Subjects then warmed up by doing 8-10 submaximal contractions at $20-80 \%$ of the estimated MVC. Following the warm-up, 3 MVCs (5-s) of the knee extensors were performed, each being superimposed by one doublet (at 100, 120 or $150 \%$ OSI) and after relaxation followed by the same intensity doublet and single twitch (with a 2 -s interval between stimulations). No more than $5 \%$ variation between MVCs was tolerated (in case of a greater variation, extra MVC(s) was/were performed); a 1-min rest period was respected between each MVC. doublet as well as potentiated doublet and twitch responses evoked at the three tested intensities. Only one example of $B$ and $C$ manoeuvers was magnified for clarity purposes. Arrows and stars, respectively, indicate where the $B$ and $C$ manoeuvers were performed. The bottom panel shows a typical record of the whole protocol

\section{Fatiguing task and post-fatigue neuromuscular assessments}

After the pre-fatigue MVCs, the first part of the fatiguing task (referred to as fatigue 1, see Fig. 1) consisted of incremental sets of 105 -s voluntary contractions (with $5 \mathrm{~s}$ rest in between) as previously used (Bachasson et al. 2013). The first set was done at a force corresponding to $10 \%$ MVC. Then, $10 \% \mathrm{MVC}$ increments were added to the following sets until task failure (i.e., inability to reach the target force for two successive contractions). A visual force feedback guided the subjects throughout the experimental protocol. In between each sets, subjects were asked to perform one 
MVC (5 s), which was followed by three doublets evoked at the three different intensities (2-s intervals between doublets); at task failure, the subjects performed three MVCs, each superimposed by one of the three intensity doublets, followed, after relaxing the muscle, by the same intensity doublet and a twitch. Three minutes of rest was allowed at this time point (referred to as post-fatigue 1). After this short recovery, subjects resumed the fatiguing task, starting one set below the former completed set. Thus, if a subject reached task failure in the middle of the $60 \%$ set during fatigue 1 , (s)he started over with the $40 \%$ set in the second part of the fatiguing task, referred to as fatigue 2 .

Data collection

All parameters were recorded on the dominant leg as determined by the revised Waterloo questionnaire (Elias et al. 1998).

\section{Evoked contractions}

A high-voltage (maximum $400 \mathrm{~V}$ ) constant-current stimulator (DS7AH, Digitimer, Hertfordshire, UK) was used to deliver single and paired electrical stimuli (pulse width: $1 \mathrm{~ms})$. The cathode $(5 \mathrm{~cm}$ diameter, Dermatrode, American Imex, Irvine, CA) and the anode $(5 \times 10 \mathrm{~cm}$, Compex, Ecublens, Switzerland) were placed over the femoral nerve at the femoral triangle level beneath the inguinal ligament and on the lower part of the gluteal fold opposite to the cathode, respectively (Neyroud et al. 2012).

The OSI level was determined by increasing the intensity until maximal twitch and $\mathrm{M}$-wave amplitudes (of the three knee extensor muscles considered). OSI was considered to be reached when an increase of 3 successive $10-\mathrm{mA}$ increments did not yield any increase in the twitch force or M-wave amplitude (Bampouras et al. 2012; RodriguezFalces et al. 2013). This intensity was then either kept constant for the stimulation at $100 \%$ OSI or increased by 20 or $50 \%$ for the stimulations at 120 and $150 \%$ OSI, i.e., supramaximal intensities.

\section{Force recordings}

Voluntary and evoked forces developed by the knee extensors were recorded using an isometric ergometer consisting of a custom-built chair equipped with a strain gauge (STS $250 \mathrm{~kg}$, sensitivity $2.0005 \mathrm{mV} / \mathrm{V}$ and $0.0017 \mathrm{~V} / \mathrm{N}$, SWJ, China). The strain gauge was attached to the chair on one end and securely strapped above the ankle with a custom made mold. Subjects were seated with a knee angle of $90^{\circ}$ and a trunk-thigh angle of $100^{\circ}\left(180^{\circ}=\right.$ full extension). Extraneous movements of the upper body were limited by two crossover shoulder harnesses and a belt across the lower abdomen. A target line on a computer screen was used as visual feedback for the target force during the fatiguing task. All force signals were recorded at $1 \mathrm{kHz}$ using an $\mathrm{AD}$ conversion system (MP150, BIOPAC, Goleta, CA).

\section{EMG recordings}

EMG activity from the knee extensors vastus lateralis $(\mathrm{VL})$, vastus medialis $(\mathrm{VM})$ and rectus femoris $(\mathrm{RF})$ as well as from the knee flexors biceps femoris (BF) was recorded using pairs of silver chloride $(\mathrm{Ag} / \mathrm{AgCl})$ circular (recording diameter of $1 \mathrm{~cm}$ ) surface electrodes (Kendall Meditrace 100, Tyco, Canada) positioned lengthwise over the middle of the muscle belly [according to SENIAM recommendations (Hermens et al. 2000)] with an interelectrode (centerto-center) distance of $2 \mathrm{~cm}$. The reference electrode was placed over the ispsilateral patella. Low resistance between the two electrodes $(<10 \mathrm{k} \Omega)$ was obtained by cleaning and lightly abrading the skin. EMG signals were amplified (gain: 1,000), filtered with a bandwidth frequency between 10 and $500 \mathrm{~Hz}$, digitized at a sampling frequency of $2 \mathrm{kHz}$ and recorded by the $\mathrm{AD}$ conversion system. Isometric force and EMG data were stored and analyzed off-line with commercially available software (AcqKnowledge software, BIOPAC Systems).

\section{Discomfort}

Discomfort associated with the three different doublets was measured after each pre-fatigue doublet (evoked prior any MVCs) using a horizontal visual analog scale $(100 \mathrm{~mm})$. Subjects, blinded to the order of stimulation intensities used, placed a vertical mark between "no discomfort" $(0 \mathrm{~mm})$ and "worst possible discomfort" (100 mm).

Data analysis

\section{Force data}

Isometric MVC forces (primary outcome) were considered as the peak force attained during the contraction. Maximal voluntary activation level (VAL) during MVCs was quantified with the superimposed and potentiated doublet amplitudes, as proposed by Allen et al. (1995). A correction was systematically applied to this equation to better estimate VAL measurement in the few cases when the superimposed doublet was elicited slightly before or after peak force (Strojnik and Komi 1998):

$$
\begin{aligned}
\mathrm{VAL}= & (1-\text { (superimposed doublet force } \\
& \times(\text { force level at stimulation } / \mathrm{MVC} \text { force }) / \\
& \text { potentiated doublet force }) \times 100
\end{aligned}
$$


Unpotentiated twitch and doublet amplitudes were analyzed to evaluate the influence of stimulation intensity on responses at rest. All further measurements were done on potentiated responses as it has been shown to be more sensitive to detect early fatigue (Kufel et al. 2002). Potentiated pre- and post-fatigue and twitch amplitudes as well as timeto-peak twitch and twitch half-relaxation time were quantified to assess contractile alterations after exercise. The amplitude of the doublets delivered at the end of each set (i.e., $10 \% \mathrm{MVC}$ set, $20 \% \mathrm{MVC}$ set, $\cdots$ ) was also analyzed to follow the time course of contractile impairment with the three stimulation intensities.

\section{EMG data}

EMG signals during the MVCs were quantified as root mean square (RMS) amplitudes for a 500-ms interval around maximum force (250-ms periods either side of the maximum force) and normalized by pre-fatigue RMS values. M-wave amplitude was measured from the single stimulation following the MVC before and after the fatiguing task. The RMS/M ratio (RMS/M-wave amplitude) was calculated as an index of muscle activation (Sale et al. 1982). $\mathrm{M}$-waves of the antagonist BF could be reliably measured in 11 out of 14 subjects.

\section{Statistical analysis}

Data normality was tested using the Kolmogorov-Smirnov test. One-way repeated measure ANOVAs (stimulation intensity) were used to test for differences between the unpotentiated twitch and associated $\mathrm{M}$-wave characteristics at the three different intensities as well as for the unpotentiated doublet amplitude. Two-way repeated measure ANOVAs (time $\times$ stimulation intensity) were used to compare different variables (potentiated peak twitch, time-to-peak twitch, half-relaxation time and doublet amplitude as well as M-wave amplitudes, MVC forces, RMS/M and VAL) between the different intensities at the different times (prefatigue, post-fatigue 1 and post-fatigue 2). Post hoc analyses (Holm-Sidak) were used to test for differences among pairs of means. The $\alpha$ level for significance was set to $p<0.05$. All statistical analyses were conducted with SigmaPlot software for Windows (version 11, Systat, Chicago, $\mathrm{IL}$, USA). Data are reported as mean \pm SD in text and tables and as mean $\pm \mathrm{SE}$ in figures.

\section{Results}

The average intensity used was $197 \pm 63 \mathrm{~mA}$ (range 70$300 \mathrm{~mA}$ ) for the $100 \%$ OSI stimulation, $237 \pm 76 \mathrm{~mA}$ (range 84-360 mA) for the $120 \%$ OSI stimulation and
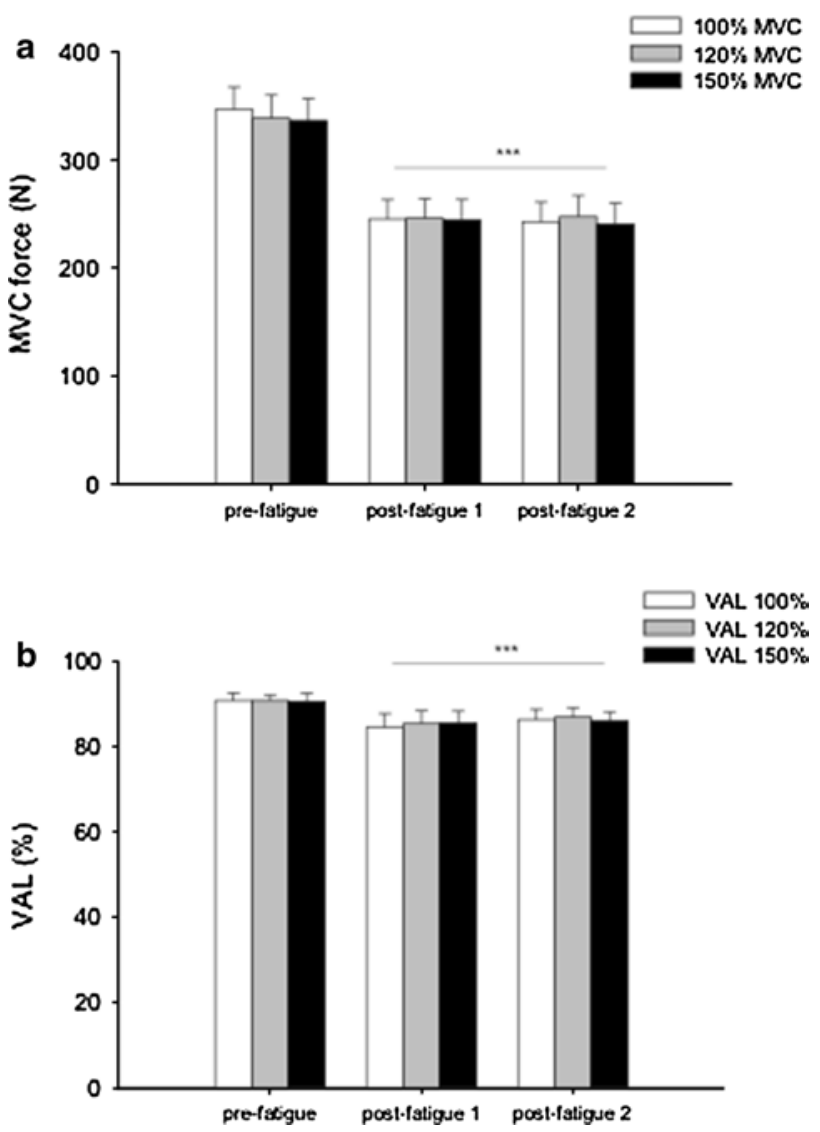

Fig. 2 Maximal voluntary contraction force (MVC, a) and voluntary activation level (VAL, b) at pre-fatigue and post-fatigue 1 and 2. $* * *$ indicates a significant difference with pre-fatigue $(p<0.001)$

$296 \pm 95 \mathrm{~mA}$ (range 105-450 mA) for the $150 \%$ OSI stimulation. The discomfort associated with the $150 \%$ OSI doublet was significantly greater than with $100 \%$ OSI $(4.5 \pm 2.3$ vs. $3.2 \pm 2.0 \mathrm{~mm}$, respectively, $p<0.05)$. The intermediate discomfort associated with the $120 \%$ OSI doublet $(3.7 \pm 2.6 \mathrm{~mm})$ was not statistically different from the other two intensities $(p>0.05)$.

MVC force and central parameters

Subjects performed an average of $64 \pm 13$ contractions during fatigue 1 . Subjects reached task failure during fatigue 2 at a similar set compared to fatigue 1 .

Pre-fatigue force levels were similar for the three stimulation intensities ( $p>0.05$, Fig. 2a). The superimposed twitch at the three different stimulation intensities was also evoked at a similar force level at task failure $(p>0.05)$. $\operatorname{VAL}(p>0.05$, Fig. 2b) and RMS/M (data not shown) were found to be similar between the three conditions; for RF only, RMS/M was significantly lower $(p<0.01)$ at $150 \%$ than at $100 \%$ OSI. 
MVC force was greatly reduced from pre- to post-fatigue $1(-30.4 \pm 11.8 \%, p<0.001$, Fig. $2 a)$, but no further decrease $(p>0.05)$ occurred between post-fatigue 1 and postfatigue $2(-32.0 \pm 10.1 \%$ when compared to pre-fatigue, Fig. 2a). RMS/M remained constant at these time points for all muscles $(p>0.05)$ and for every OSI considered. VAL decreased by about $5 \%$ post-fatigue 1 (from $90.6 \pm 6.3$ to $85.0 \pm 10.9 \%, p<0.01$, Fig. 2b) with no further reduction $(p>0.05)$ observed at post-fatigue $2(86.3 \pm 8.9 \%)$.

\section{M-wave amplitude}

No effect of stimulation intensity was found on VL unpotentiated M-waves $(p>0.05)$, whereas VM $(p<0.01)$ and RF $(p<0.02)$ M-wave amplitudes were greater when evoked at $150 \%$ OSI compared to $100 \%$. Similar to the unpotentiated responses, no influence of stimulation intensity was found for potentiated VL M-wave amplitude $(p>0.05$, Table 1$)$. However, potentiated VM $(p<0.01)$ and RF $(p<0.001) \mathrm{M}$-wave amplitudes were higher when the stimulation was evoked at $150 \%$ compared to $100 \%$ OSI, both before and after the fatiguing task. Further, the potentiated M-wave amplitude for RF evoked at $120 \%$ OSI was also significantly greater than the potentiated $100 \%$ OSI one $(p<0.05)$. Potentiated VM $(p>0.05)$ and RF $(p>0.05) \mathrm{M}$-wave amplitudes evoked at $120 \%$ OSI were similar to $150 \%$ OSI. BF M-wave amplitude increased while knee extensors were stimulated at $150 \%$ compared to $100 \%$ OSI $(n=11, p<0.001$, Table 1$)$.

The fatiguing task exercise induced a reduction $(\sim 7 \%)$ in VM M-wave amplitude at all stimulation intensities $(p<0.01$, Table 1$)$. M-wave amplitudes from the other muscles remained unchanged after exercise $(p>0.05$, Table 1).

\section{Contractile properties}

Prior to any MVCs in the resting state, the unpotentiated responses showed a slightly greater evoked force when using $150 \%$ OSI intensity compared to $100 \%$ OSI $(56.0 \pm 13.5 \mathrm{~N}$ vs. $54.4 \pm 13.3 \mathrm{~N}, p<0.01)$. The $120 \%$ OSI unpotentiated twitch $(55.3 \pm 13.2 \mathrm{~N})$ did not differ from $100 \%(p>0.05)$ or $150 \%$ OSI $(p>0.05)$. Furthermore, unpotentiated doublet forces were similar between the three intensities at pre-fatigue $(100 \%: 95.3 \pm 20.4 \mathrm{~N}$, $120 \%$ : $95.7 \pm 20.8 \mathrm{~N}$ and $150 \%: 96.5 \pm 19.7 \mathrm{~N}, p>0.05$ ).

Thereafter, when the potentiated responses were considered, stimulation intensity did not significantly affect pre- or post-fatigue potentiated twitch or doublet amplitudes ( $p>0.05$, see Fig. 3). Time-to-peak twitch and twitch half-relaxation time were also not influenced by stimulation intensity, whatever the time point considered (data not shown, $p>0.05$ ).
Table 1 M-wave amplitude at 100, 120 and $150 \%$ of optimal stimulation intensity before and after the two fatiguing exercises

\begin{tabular}{|c|c|c|c|}
\hline \multirow[t]{2}{*}{ Intensity } & \multicolumn{3}{|c|}{ Amplitude (mV) } \\
\hline & Pre-fatigue & Post-fatigue 1 & Post-fatigue 2 \\
\hline \multicolumn{4}{|l|}{ VL } \\
\hline $100 \%$ & $8.6 \pm 3.7$ & $8.7 \pm 3.6$ & $8.1 \pm 3.6$ \\
\hline $120 \%$ & $8.8 \pm 3.8$ & $8.9 \pm 3.3$ & $8.5 \pm 3.5$ \\
\hline $150 \%$ & $8.7 \pm 3.6$ & $8.9 \pm 3.2$ & $8.4 \pm 3.4$ \\
\hline \multicolumn{4}{|l|}{ VM } \\
\hline $100 \%$ & $13.1 \pm 5.1$ & $12.6 \pm 5.9$ & $11.6 \pm 5.7^{* *}$ \\
\hline $120 \%$ & $13.9 \pm 4.9$ & $13.5 \pm 5.3$ & $12.6 \pm 5.1^{* * *}$ \\
\hline $150 \%$ & $14.3 \pm 4.4^{\dagger \dagger}$ & $14.0 \pm 4.7^{\dagger \dagger}$ & $13.2 \pm 4.5^{* *, \dagger}$ \\
\hline \multicolumn{4}{|l|}{ RF } \\
\hline $100 \%$ & $7.3 \pm 2.2$ & $7.3 \pm 2.5$ & $7.3 \pm 2.4$ \\
\hline $120 \%$ & $7.7 \pm 2.3^{\dagger}$ & $7.7 \pm 2.4^{\dagger}$ & $7.8 \pm 2.2^{\dagger}$ \\
\hline $150 \%$ & $8.0 \pm 2.4^{\dagger}$ & $7.9 \pm 2.3^{\dagger}$ & $8.0 \pm 2.3^{\dagger}$ \\
\hline \multicolumn{4}{|l|}{$\mathrm{BF}$} \\
\hline $100 \%$ & $3.4 \pm 1.8$ & $3.5 \pm 2.4$ & $3.4 \pm 2.4$ \\
\hline $120 \%$ & $3.9 \pm 2.3$ & $4.2 \pm 2.6$ & $4.1 \pm 2.8$ \\
\hline $150 \%$ & $4.5 \pm 2.4^{\dagger \dagger}$ & $4.5 \pm 2.9^{\dagger \dagger}$ & $4.5 \pm 3.1^{\dagger \dagger}$ \\
\hline
\end{tabular}

Data are presented as mean \pm SD

$n=14$ for the vastus lateralis (VL), vastus medialis (VM) and rectus femoris (RF) and $n=11$ for the antagonist biceps femoris (BF)

** indicates a significant difference compared to pre-fatigue $(p<0.01)$

$\dagger$ and ${ }^{\dagger}$ indicates significant differences compared to the M-wave evoked at $100 \%$ of optimal stimulation intensity $(p<0.05$ and $p<0.01$, respectively)

At post-fatigue 1, potentiated twitch and doublet amplitudes were diminished $(-31.0 \pm 21.9 \%$ for twitch amplitude and $-23.4 \pm 18.9 \%$ for doublet amplitude, $p<0.001$ ) for all the considered intensities (Fig. 3). A trend towards a decrease in doublet amplitude was observed between post-fatigue 1 and post-fatigue 2 $(-9.1 \%, p=0.075)$. When considering the peak twitch amplitude, no significant decrease was found between post-fatigue 1 and post-fatigue 2 for 100 and $120 \%$ OSI, although statistical trends were found $(-12.1 \pm 11.4 \%$ for $100 \%$ OSI, $p=0.089$ and $-10.8 \pm 9.4 \%$ for $120 \%$ OSI, $p=0.103$ when comparing post-fatigue 2 to postfatigue 1 values, respectively). Meanwhile, a significant decrease was observed for the $150 \%$ OSI twitch amplitude $(-16.6 \pm 10.4 \%, p<0.05)$.

MVC and doublet force time course through fatigue 1

Due to the wide differences in the number of sets completed across subjects (range 40-100\% MVC set) and in order to keep a sufficient number of included subjects for the statistical analysis, we only considered forces 

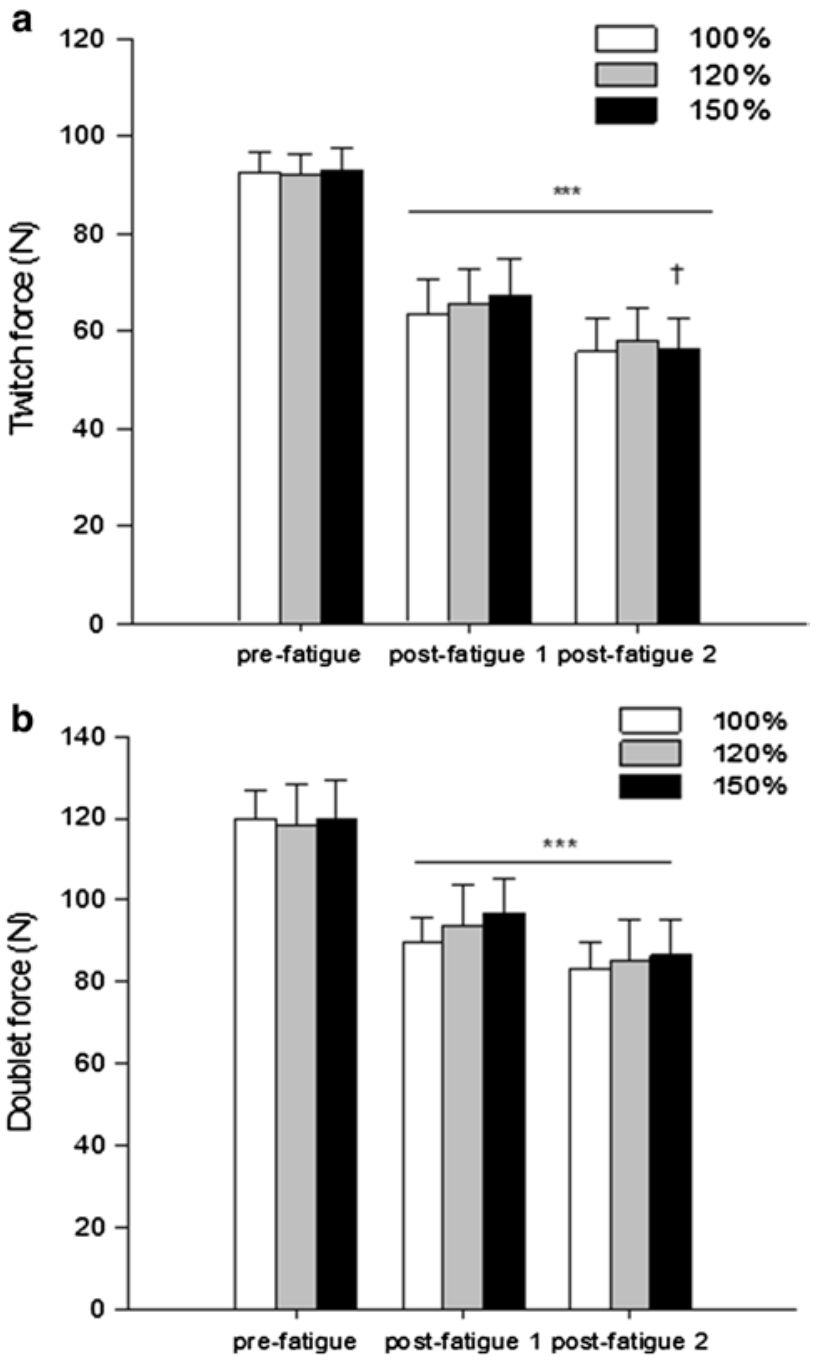

Fig. 3 Potentiated twitch (a) and potentiated doublet (b) forces at pre-fatigue and post-fatigue 1 and 2 . $* * *$ indicates a significant difference with pre-fatigue $(p<0.001),{ }^{\dagger}$ indicates a significant difference with post-fatigue $1(p<0.05)$

until post-60 \% MVC set $(n=10)$. MVC force was significantly reduced $(p<0.001)$ from the set performed at $20 \%$ MVC. Additionally, doublet amplitude was reduced after the set performed at $60 \% \operatorname{MVC}(p<0.001)$. It also appeared that the doublet amplitudes after the $40 \%$ and $50 \%$ MVC sets were greater when assessed with the stimulation at 120 or $150 \%$ OSI compared to $100 \%$ OSI $(p<0.05)$

\section{Discussion}

The present study was designed to determine the stimulation intensity necessary for an adequate assessment of central and peripheral components of neuromuscular fatigue of the knee extensors. With this aim, we induced fatigue of the knee extensors and applied three different stimulation intensities. We successfully induced both peripheral alterations (as indicated by the decreased twitch and doublet amplitudes) and, to a lesser extent, central impairments (as indicated by the reduced VAL). As hypothesized, we found that stimulating at $100 \%$ OSI was not enough to ensure maximal recruitment of all motor units in the fatigued state; the use of this stimulation intensity may thus overestimate the extent of peripheral fatigue. Also in agreement with our hypothesis, both greater discomfort and greater antagonist co-activation were associated with stimulating at $150 \%$ OSI. It follows that for the assessment of knee extensor fatigue characteristics, a stimulation intensity $>100$ but $<150 \%$ OSI should be used.

\section{Induced fatigue}

Our fatigue protocol, comprising two task failure points, induced a large amount of neuromuscular fatigue, as highlighted by the MVC force loss ( 30\%). Recently, Bachasson et al. (2013) found a similar force reduction after an identical incremental fatiguing task to exhaustion. Strikingly, in our study, no further MVC force decrease was observed post-fatigue 2 . This finding is in agreement with the notion of neuromuscular reserve brought up by several authors (Swart et al. 2009; Marcora and Staiano 2010; Millet 2011; Amann 2011; Neyroud et al. 2013). The MVC force loss we observed can be attributed to a large extent to contractile impairments. For all tested intensities, at post-fatigue 1 , doublet responses were reduced by about $23 \%$ and twitches by about $31 \%$, whereas VAL was only decreased by $5 \%$ and RMS/M was unchanged. These results are in accordance with the study of Bachasson et al. (Bachasson et al. 2013) who also found important peripheral impairments, as reflected by substantial twitch and doublet reductions at exhaustion $(-41$ and $-37 \%$ of pre-fatigue values for twitch and doublet, respectively) and a small central impairment highlighted by a $2 \% \mathrm{VAL}$ decrease.

Which stimulation intensity to assess neuromuscular fatigue?

In the literature, stimulation intensities ranging from $100 \%$ OSI (Babault et al. 2001; Place et al. 2005; Wüst et al. 2008) to $150 \%$ OSI (Löscher et al. 1996; Lagerquist and Collins 2010; Millet et al. 2011b), including intensities of $110 \%$ (Babault et al. 2001; Adam and De Luca 2005; Zory et al. 2005; Neyroud et al. 2013), $120 \%$ (Löscher et al. 1996; Gondin et al. 2006; Requena et al. 2008; Sidhu et al. 2009; Perrey et al. 2010), $125 \%$ (Hicks et al. 1989), and $130 \%$ (Klass et al. 2012; Froyd et al. 2013) OSI have been used to study muscle fatigue. 
Additionally, it should be kept in mind that nerve stimulation presents some limitations such as the induced discomfort and antagonist co-activation (Place et al. 2010a). We, therefore, investigated whether discrepancies would be observed with different stimulation intensities when assessing neuromuscular function at rest, during and immediately after a fatiguing exercise.

Our results show that for the assessment of VAL similar results are obtained, whatever the intensity of stimulation used. In their study, Folland and Williams (2007) assessed VAL by superimposing either a small twitch (eliciting $12 \%$ MVC), a large twitch (eliciting $20 \%$ MVC) or a doublet (eliciting $30 \% \mathrm{MVC}$ ) evoked over knee extensors and also found no significant difference in the VAL obtained. Bampouras et al. (2012) also found that twitch interpolation using paired stimulations delivered over the knee extensors, at submaximal intensity ( $>50 \%$ of the intensity eliciting a plateau in the evoked force) resulted in similar VAL as compared to stimulating at $100 \%$. Similar results were also reported by others (Rutherford et al. 1986; Martin et al. 2004). In summary, it appears that for both un-fatigued and fatigued states, high supramaximal stimulation intensity is not needed for VAL assessment of MVCs for the knee extensors.

Similar to VAL, doublet evoked responses were similar across the different intensities of stimulation before and after exercise. This suggests that $100 \%$ OSI would be high enough to yield consistent and reliable results. However, during exercise (here post $-40 \%$ and post-50 \% MVC sets), when both potentiation and fatigue occur (Rassier and Macintosh 2000), the $100 \%$ OSI doublet produced a smaller evoked force compared to the 120 and $150 \%$ OSI doublets. Similarly, an intensity effect was found when considering the twitch responses. Indeed, with fatigue, the motor unit axonal excitation threshold increases (Kernell and Monster 1982a, b; Vagg et al. 1998; Butler et al. 2003) and, therefore, $100 \%$ OSI might not be enough anymore to recruit $100 \%$ of motor units.

Regarding the twitch responses, even though no intensity effect was observed in the unfatigued state for potentiated responses, the unpotentiated twitch evoked at $150 \%$ OSI induced a slightly $(<3 \%)$ greater force than the one evoked at $100 \%$ OSI. At first glance, our data indicate that motor unit recruitment was complete at $100 \%$ OSI as no difference was seen between 100 and $120 \%$ OSI. As the level of coactivation, assessed with M-wave amplitude of the biceps femoris muscle, was higher at $150 \%$ when compared to $100 \%$ OSI, the higher values obtained at $150 \%$ OSI cannot be attributed to reduced antagonist activation. We can also discard a possible voluntary contribution because of increased discomfort, as (i) no voluntary EMG was present right after the stimulation at any of the three stimulation intensities, and (ii) the shape of the twitch was similar at each stimulation intensity. Thus, the most plausible explanation for the difference observed between 100 and $150 \%$ OSI (which also holds for M-wave amplitude of $\mathrm{VM}$ and RF muscles) is that a few motor units were not recruited at $100 \%$ OSI. It is interesting to note that a $50 \%$ increase in the stimulation intensity was necessary to further recruit these presumably silent motor units. Anyway, these results favor the use of the potentiated response in the assessment of neuromuscular fatigue, in agreement with a previous study (Kufel et al. 2002) reporting a greater reproducibility of potentiated twitches than unpotentiated ones. It should be noted that stimulation intensity appeared to be less important if the main interest resides in the temporal properties of the twitch, as no differences were observed in time-to-peak twitch or half-relaxation rate across intensities.

Alongside with these mechanical alterations, an intensity main effect was found for M-wave amplitude. Both in the fresh and fatigued state, VM and RF muscles showed greater potentiated and unpotentiated responses when stimulated at $150 \%$ OSI. These greater unpotentiated M-wave amplitudes $(+12.4 \%$ for VM and $+8.3 \%$ for RF when comparing 150-100\% OSI) highlight the difficulty of defining and determining OSI in the knee extensors and presumably also in other muscle groups comprising several muscle heads. In practice (and as done in the present study), most researchers determine a plateau in peak twitch by increasing stimulation intensity by one to four $10 \mathrm{~mA}$ increments (Bampouras et al. 2012; Rodriguez-Falces et al. 2013), but not always checking for a plateau in M-wave amplitude. Our results suggest that relying on peak twitch amplitude might not be sufficient to accurately determine a "true" OSI. In our set-up, determining a "true" OSI would have necessitated an intensity increase by about 10 increments to reach $150 \%$ OSI. It is unlikely that researchers will increase the current intensity by an average further $100 \mathrm{~mA}$ once they reach what they consider OSI. We, therefore, chose to determine the OSI as usually done in practice without checking that $150 \%$ OSI led to a similar response as $100 \%$ OSI. A practical recommendation based on our findings would be to verify that both twitch and M-wave amplitudes evoked at least at $120 \%$ OSI are similar to those at $100 \%$. Furthermore, we would like to highlight the finding that even though unpotentiated $150 \%$ OSI responses were greater than $100 \%$ OSI, this difference disappeared when the potentiated responses, which have been recommended for fatigue assessment (Kufel et al. 2002; Place et al. 2007), were considered. We would also like to emphasize that the present results apply to knee extensors and that further studies would be required for other muscle groups.

If at first glance it would seem 'safe' to use a further increase in stimulation intensity, one has to keep in mind 
that this will come with a possible parallel increase in coactivation of antagonist muscles, which would impact the mechanical response, even though other factors such as electrode size and position might also impact antagonist co-activation. In our set-up, activation of the antagonist $\mathrm{BF}$ muscle was increased at $150 \%$ OSI by about $30 \%$, whereas $120 \%$ OSI did not result in a greater co-activation than $100 \%$. The optimal intensity should, therefore, be a compromise to reduce the possible contribution of the antagonist muscle to the mechanical response. In some setups, twitch amplitude and M-wave responses are recorded separately, for instance in the elbow flexors (Neyroud et al. 2013). In such cases, $150 \%$ OSI could be used to record M-waves, whereas only $120 \%$ would be enough for the mechanical response.

A greater discomfort with $150 \%$ OSI was found compared to $100 \%$ OSI, whereas the discomfort associated with $120 \%$ OSI was not statistically different from the two other intensities. Discomfort of stimulation can be minimized by varying several other factors than current intensity, such as stimulation location, electrode size and/ or pulse width; a pulse width of $1 \mathrm{~ms}$ was chosen to allow comparison with previous results obtained in our laboratory and others (Place et al. 2010a; Neyroud et al. 2012, 2013; Rupp et al. 2012; Temesi et al. 2013). Minimizing pain/ discomfort is of interest as expectation of noxious stimuli might lead to reduced maximum voluntary force and thus biased measurements (Button and Behm 2008), even though no difference in MVC force was found between the three intensities used in the present study. Moreover, it has been found that in some patients with knee osteoarthritis, increase in the stimulation intensity from 100 to $120 \%$ OSI was not possible due to discomfort (Staehli et al. 2010). These results indicate the necessity to minimize the discomfort associated with the stimulation to decrease subjects' apprehension and to allow the use of stimulation in a clinical population.

\section{Conclusions}

On one hand, our results show that using $100 \%$ OSI does not guarantee complete recruitment and may, therefore, lead to overestimation of peripheral fatigue levels. On the other hand, $150 \%$ OSI leads to greater co-activation and more discomfort compared to $100 \%$ OSI. Additionally, 100, 120 and $150 \%$ OSI led to similar VAL, doublet and potentiated twitch forces. Thus, $120 \%$ OSI appears to represent the intensity of choice to assess neuromuscular fatigue for the quadriceps as it does not induce greater co-activation and discomfort than $100 \%$ OSI and does not elicit different mechanical and electrical responses than $150 \%$ OSI. We, therefore, recommend using $120 \%$ OSI, as it constitutes a good trade-off between discomfort and reliable measurements.

Acknowledgments This study was supported by the De Reuter foundation and the Geneva Academic Society. The authors thank all the subjects who volunteered to participate and Marc Buclin for the design and conception of the ergometer.

Conflict of interest The authors declare that they have no conflict of interest.

\section{References}

Adam A, De Luca CJ (2005) Firing rates of motor units in human vastus lateralis muscle during fatiguing isometric contractions. $\mathrm{J}$ Appl Physiol 99(1):268-280

Allen GM, Gandevia SC, McKenzie DK (1995) Reliability of measurements of muscle strength and voluntary activation using twitch interpolation. Muscle Nerve 18(6):593-600

Alon G (1985) High voltage stimulation. Effects of electrode size on basic excitatory responses. Phys Ther 65(6):890-895

Amann M (2011) Central and peripheral fatigue: interaction during cycling exercise in humans. Med Sci Sports Exerc 43(11):2039-2045

Babault N, Pousson M, Ballay Y, Van Hoecke J (2001) Activation of human quadriceps femoris during isometric, concentric, and eccentric contractions. J Appl Physiol 91(6):2628-2634

Bachasson D, Millet GY, Decorte N, Wuyam B, Levy P, Verges S (2013) Quadriceps function assessment using an incremental test and magnetic neurostimulation: a reliability study. J Electromyogr Kinesiol 23(3):649-658

Bampouras TM, Reeves ND, Baltzopoulos V, Jones DA, Maganaris CN (2012) Is maximum stimulation intensity required in the assessment of muscle activation capacity? J Electromyogr Kinesiol 22(6):873-877

Behm DG, St-Pierre DM, Perez D (1996) Muscle inactivation: assessment of interpolated twitch technique. J Appl Physiol 81:2267-2273

Bigland-Ritchie B, Furbush F, Woods JJ (1986) Fatigue of intermittent submaximal voluntary contractions: central and peripheral factors. J Appl Physiol 61(2):421-429

Burke D (2002) Effects of activity on axonal excitability: implications for motor control studies. Adv Exp Med Biol 508:33-37

Butler JE, Taylor JL, Gandevia SC (2003) Responses of human motoneurons to corticospinal stimulation during maximal voluntary contractions and ischemia. J Neurosci 23(32): 10224-10230

Button DC, Behm DG (2008) The effect of stimulus anticipation on the interpolated twitch technique. J Sports Sci Med 7:520-524

Edwards RH, Hill DK, Jones DA, Merton PA (1977) Fatigue of long duration in human skeletal muscle after exercise. J Physiol 272(3):769-778

Elias LJ, Bryden MP, Bulman-Fleming MB (1998) Footedness is a better predictor than is handedness of emotional lateralization. Neuropsychologia 36(1):37-43

Folland JP, Williams AG (2007) Methodological issues with the interpolated twitch technique. J Electromyogr Kinesiol 17(3): 317-327

Froyd C, Millet GY, Noakes TD (2013) The development of peripheral fatigue and short-term recovery during self-paced high-intensity exercise. J Physiol 591:1339-1346

Gandevia SC (2001) Spinal and supraspinal factors in human muscle fatigue. Physiol Rev 81(4):1725-1789 
Gondin J, Guette M, Jubeau M, Ballay Y, Martin A (2006) Central and peripheral contributions to fatigue after electrostimulation training. Med Sci Sports Exerc 38(6):1147-1156

Hamada T, Sale DG, MacDougall JD, Tarnopolsky MA (2000) Postactivation potentiation, fiber type, and twitch contraction time in human knee extensor muscles. J Appl Physiol 88(6): 2131-2137

Hermens HJ, Freriks B, Disselhorst-Klug C, Rau G (2000) Development of recommendations for SEMG sensors and sensor placement procedures. J Electromyogr Kinesiol 10(5):361-374

Hicks A, Fenton J, Garner S, McComas AJ (1989) M wave potentiation during and after muscle activity. J Appl Physiol 66(6):2606-2610

Kernell D, Monster AW (1982a) Time course and properties of late adaptation in spinal motoneurones of the cat. Exp Brain Res 46(2):191-196

Kernell D, Monster AW (1982b) Motoneurone properties and motor fatigue. An intracellular study of gastrocnemius motoneurones of the cat. Exp Brain Res 46(2):197-204

Klass M, Roelands B, Levenez M, Fontenelle V, Pattyn N, Meeusen R, Duchateau J (2012) Effects of noradrenaline and dopamine on supraspinal fatigue in well-trained men. Med Sci Sports Exerc 44(12):2299-2308

Kooistra RD, de Ruiter CJ, de Haan A (2007) Conventionally assessed voluntary activation does not represent relative voluntary torque production. Eur J Appl Physiol 100(3):309-320

Kubo K, Tsunoda N, Kanehisa H, Fukunaga T (2004) Activation of agonist and antagonist muscles at different joint angles during maximal isometric efforts. Eur J Appl Physiol 91(2-3):349-352

Kufel TJ, Pineda LA, Mador MJ (2002) Comparison of potentiated and unpotentiated twitches as an index of muscle fatigue. Muscle Nerve 25(3):438-444

Lagerquist O, Collins DF (2010) Influence of stimulus pulse width on $\mathrm{M}$-waves, $\mathrm{H}$-reflexes, and torque during tetanic low-intensity neuromuscular stimulation. Muscle Nerve 42(6):886-893

Lévénez M, Kotzamanidis C, Carpentier A, Duchateau J (2005) Spinal reflexes and coactivation of ankle muscles during a submaximal fatiguing contraction. J Appl Physiol 99:1182-1188

Löscher WN, Cresswell AG, Thorstensson A (1996) Central fatigue during a long-lasting submaximal contraction of the triceps surae. Exp Brain Res 108(2):305-314

Marcora SM, Staiano W (2010) The limit to exercise tolerance in humans: mind over muscle? Eur J Appl Physiol 109(4): 763-770

Martin V, Millet GY, Martin A, Deley G, Lattier G (2004) Assessment of low-frequency fatigue with two methods of electrical stimulation. J Appl Physiol 97(5):1923-1929

Merton PA (1954) Voluntary strength and fatigue. J Physiol 123(3):553-564

Millet GY (2011) Can neuromuscular fatigue explain running strategies and performance in ultra-marathons?: the flush model. Sports Med 41(6):489-506

Millet GY, Lepers R (2004) Alterations of neuromuscular function after prolonged running, cycling and skiing exercises. Sports Med 34(2):105-116

Millet GY, Martin V, Martin A, Vergès S (2011a) Electrical stimulation for testing neuromuscular function: from sport to pathology. Eur J Appl Physiol 111(10):2489-2500

Millet GY, Tomazin K, Verges S, Vincent C, Bonnefoy R, Boisson RC, Gergele L, Feasson L, Martin V (2011b) Neuromuscular consequences of an extreme mountain ultra-marathon. PLoS ONE 6(2):1-14

Millet GY, Bachasson D, Temesi J, Wuyam B, Feasson L, Verges S, Levy P (2012) Potential interests and limits of magnetic and electrical stimulation techniques to assess neuromuscular fatigue. Neuromuscul Disord 22(Suppl 3):S181-S186
Neyroud D, Maffiuletti NA, Kayser B, Place N (2012) Mechanisms of Fatigue and Task Failure Induced By Sustained Submaximal Contractions. Med Sci Sports Exerc 44:1243-1251

Neyroud D, Ruttimann J, Mannion AF, Millet GY, Maffiuletti NA, Kayser B, Place N (2013) Comparison of neuromuscular adjustments associated with sustained isometric contractions of four different muscle groups. J Appl Physiol 114:1426-1434

Perrey S, Racinais S, Saimouaa K, Girard O (2010) Neural and muscular adjustments following repeated running sprints. Eur J Appl Physiol 109(6):1027-1036

Place N, Maffiuletti NA, Ballay Y, Lepers R (2005) Twitch potentiation is greater after a fatiguing submaximal isometric contraction performed at short vs. long quadriceps muscle length. J Appl Physiol 98(2):429-436

Place N, Maffiuletti NA, Martin A, Lepers R (2007) Assessment of the reliability of central and peripheral fatigue after sustained maximal voluntary contraction of the quadriceps muscle. Muscle Nerve 35:486-495

Place N, Casartelli N, Glatthorn JF, Maffiuletti NA (2010a) Comparison of quadriceps inactivation between nerve and muscle stimulation. Muscle Nerve 42(6):894-900

Place N, Yamada T, Bruton JD, Westerblad H (2010b) Muscle fatigue: from observations in humans to underlying mechanisms studied in intact single muscle fibres. Eur J Appl Physiol 110:1-15

Rassier DE, Macintosh BR (2000) Coexistence of potentiation and fatigue in skeletal muscle. Braz J Med Biol Res 33(5):499-508

Requena B, Gapeyeva H, Garcia I, Ereline J, Paasuke M (2008) Twitch potentiation after voluntary versus electrically induced isometric contractions in human knee extensor muscles. Eur J Appl Physiol 104(3):463-472

Rodriguez-Falces J, Maffiuletti NA, Place N (2013) Twitch and M-wave potentiation induced by intermittent maximal voluntary quadriceps contractions: Differences between direct quadriceps and femoral nerve stimulation. Muscle Nerve. doi: $10.1002 /$ mus. 23856

Rupp T, Jubeau M, Wuyam B, Perrey S, Levy P, Millet GY, Verges S (2012) Time-dependent effect of acute hypoxia on corticospinal excitability in healthy humans. J Neurophysiol 108(5):1270-1277

Rutherford OM, Jones DA, Newham DJ (1986) Clinical and experimental application of the percutaneous twitch superimposition technique for the study of human muscle activation. J Neurol Neurosurg Psychiatry 49(11):1288-1291

Sale D, Quinlan J, Marsh E, McComas AJ, Belanger AY (1982) Influence of joint position on ankle plantarflexion in humans. J Appl Physiol 52(6):1636-1642

Sidhu SK, Bentley DJ, Carroll TJ (2009) Locomotor exercise induces long-lasting impairments in the capacity of the human motor cortex to voluntarily activate knee extensor muscles. J Appl Physiol 106(2):556-565

Sogaard K, Gandevia SC, Todd G, Petersen NT, Taylor JL (2006) The effect of sustained low-intensity contractions on supraspinal fatigue in human elbow flexor muscles. J Physiol 573:511-523

Staehli S, Glatthorn JF, Casartelli N, Maffiuletti NA (2010) Testretest reliability of quadriceps muscle function outcomes in patients with knee osteoarthritis. J Electromyogr Kinesiol 20(6):1058-1065

Strojnik V, Komi PV (1998) Neuromuscular fatigue after maximal stretch-shortening cycle exercise. J Appl Physiol 84(1):344-350

Swart J, Lamberts RP, Lambert MI, Lambert EV, Woolrich RW, Johnston S, Noakes TD (2009) Exercising with reserve: exercise regulation by perceived exertion in relation to duration of exercise and knowledge of endpoint. Br J Sports Med 43(10):775-781

Temesi J, Arnal PJ, Davranche K, Bonnefoy R, Levy P, Verges S, Millet GY (2013) Does Central Fatigue Explain Reduced Cycling after Complete Sleep Deprivation? Med Sci Sports Exerc. doi:10. 1249/MSS.0b013e31829ce379 
Vagg R, Mogyoros I, Kiernan MC, Burke D (1998) Activity-dependent hyperpolarization of human motor axons produced by natural activity. J Physiol 507:919-925

Wüst RC, Morse CI, de Haan A, Rittweger J, Jones DA, Degens H (2008) Skeletal muscle properties and fatigue resistance in relation to smoking history. Eur J Appl Physiol 104(1):103-110
Zory R, Boerio D, Jubeau M, Maffiuletti NA (2005) Central and peripheral fatigue of the knee extensor muscles induced by electromyostimulation. Int J Sports Med 26(10):847-853 